\title{
Monitoring batch processes with dynamic time warping and k-nearest neighbours
}

\author{
Spooner, Max; Kulahci, Murat
}

Published in:

Chemometrics and Intelligent Laboratory Systems

Link to article, DOI:

10.1016/j.chemolab.2018.10.011

Publication date:

2018

Document Version

Peer reviewed version

Link back to DTU Orbit

Citation $(A P A)$ :

Spooner, M., \& Kulahci, M. (2018). Monitoring batch processes with dynamic time warping and k-nearest neighbours. Chemometrics and Intelligent Laboratory Systems, 183, 102-112.

https://doi.org/10.1016/j.chemolab.2018.10.011

\section{General rights}

Copyright and moral rights for the publications made accessible in the public portal are retained by the authors and/or other copyright owners and it is a condition of accessing publications that users recognise and abide by the legal requirements associated with these rights.

- Users may download and print one copy of any publication from the public portal for the purpose of private study or research.

- You may not further distribute the material or use it for any profit-making activity or commercial gain

- You may freely distribute the URL identifying the publication in the public portal

If you believe that this document breaches copyright please contact us providing details, and we will remove access to the work immediately and investigate your claim. 


\section{Accepted Manuscript}

Monitoring batch processes with dynamic time warping and k-nearest neighbours

Max Spooner, Murat Kulahci

PII: S0169-7439(18)30200-4

DOI: $\quad$ https://doi.org/10.1016/j.chemolab.2018.10.011

Reference: CHEMOM 3697

To appear in: Chemometrics and Intelligent Laboratory Systems

Received Date: 6 April 2018

Revised Date: 24 October 2018

Accepted Date: 26 October 2018

Please cite this article as: M. Spooner, M. Kulahci, Monitoring batch processes with dynamic time warping and k-nearest neighbours, Chemometrics and Intelligent Laboratory Systems (2018), doi: https://doi.org/10.1016/j.chemolab.2018.10.011.

This is a PDF file of an unedited manuscript that has been accepted for publication. As a service to our customers we are providing this early version of the manuscript. The manuscript will undergo copyediting, typesetting, and review of the resulting proof before it is published in its final form. Please note that during the production process errors may be discovered which could affect the content, and all legal disclaimers that apply to the journal pertain. 


\title{
Monitoring batch processes with dynamic time warping and k-nearest neighbours
}

\author{
Max Spooner ${ }^{\mathrm{b}, *}$, Murat Kulahci ${ }^{\mathrm{a}, \mathrm{b}}$ \\ ${ }^{a}$ DTU Compute, Technical University of Denmark, Kgs. Lyngby, Denmark \\ ${ }^{b}$ Department of Business Administration, Technology and Social Sciences, Lulea University \\ of Technology, Luleå, Sweden
}

\begin{abstract}
A novel data driven approach to batch process monitoring is presented, which combines the k-Nearest Neighbour rule with the dynamic time warping (DTW) distance. This online method (DTW-NN) calculates the DTW distance between an ongoing batch, and each batch in a reference database of batches produced under normal operating conditions (NOC). The sum of the $k$ smallest DTW distances is monitored. If a fault occurs in the ongoing batch, then this distance increases and an alarm is generated. The monitoring statistic is easy to interpret, being a direct measure of similarity of the ongoing batch to its nearest NOC predecessors and the method makes no distributional assumptions regarding normal operating conditions. DTW-NN is applied to four extensive datasets from simulated batch production of penicillin, and tested on a wide variety of fault types, magnitudes and onset times. Performance of DTW-NN is contrasted with a benchmark multiway PCA approach, and DTW-NN is shown to perform particularly well when there is clustering of batches under NOC.
\end{abstract}

Keywords: batch process, dynamic time warping, nearest neighbours, pensim

\section{Introduction}

Batch processes are a widespread method of production in the manufacturing, food and medicine industries among others. In batch processes, quantities

\footnotetext{
* Corresponding author

Email address: mpsp@dtu.dk (Max Spooner)
} 
of raw materials are subjected to a sequence of steps and conditions over a finite

5 duration to transform them into the final product. Successful batches should meet product specifications and display as little variation as possible, and statistical process monitoring (SPM) is a useful tool for facilitating this goal. The data produced by a batch process has a three-way structure, consisting of $I$ batches, for each of which $J$ variables are measured at $N$ time points throughout the batch's duration. Given this data, it is the goal of SPM to identify when a batch is out of control, .

Each mode of this data structure poses challenges to the application of SPM including high dimensionality in the variable mode (large $J$ ), with correlation between variables, different durations and rates of progress for different batches

15 (varying $N$ ), the non-stationary and non-linear nature of the variable trajectories, changing covariance structure throughout a batch, and clustering of batches according to, for example, changes in raw materials, or maintenance schedules.

Nomikos and MacGregor [1] pioneered the application of SPM to batch processes with their development of the multiway principal component analysis (MPCA) monitoring scheme. This method deals with the three way data structure through unfolding such that the time dimension is combined with the variable dimension (often referred to as batch-wise unfolding). A PCA model is built on the unfolded data from a set of historical batches known to represent normal operating conditions (NOC). The model can then be used online during future batches, in order to detect deviations from NOC behaviour. Wold et al. 2] proposed a method of unfolding (dubbed variable-wise unfolding) whereby the batch dimension is combined with the time dimension prior to PCA model fitting and monitoring. However, this approach assumes, unrealistically, the same correlation structure between variables at every time-point in the process $[3$, and so so the batch-wise unfolding approach [1] is adopted as the benchmark method in this work.

In 1, batches of equal duration were assumed and are required for the MPCA method. Subsequently, the issue of how to deal with variation in batch duration was widely investigated, leading to a number of different methods for 
aligning batch process data. One approach made use of a so-called indicator variable, and re-sampled the data at even increments of this variable to obtain batches of the same length [4,5]. In the chemometrics field, correlation optimised warping (COW) was developed for correcting peak-shifts in chromatographic data [6], and this method was later applied to aligning batch process data [7.

40 Another alignment method known as dynamic time warping (DTW) was applied to batch process SPM by Kassidas et al. 8]. This technique can synchronise trajectories of different lengths, and provides a distance measure quantifying the similarity of the trajectories. Online application of DTW is especially challenging as it entails aligning an incomplete batch to the most appropriate sub-section of a completed batch. Adaptations to the DTW algorithm have been proposed [9, 10] which aim to improve performance of online-alignment by limiting the degree of permitted re-alignment at each time-point. Other authors have proposed methods which avoid explicit synchronisation of the data, for example by applying feature extraction [11. In this work, the distance measure provided by DTW is used to quantify an ongoing batch's distance to NOC batches so that the DTW alignment itself is the basis of the monitoring framework. As the effect of alternative DTW implementations such as [9, 10] on the DTW distance is uncertain, the classic DTW algorithm as originally introduced to batch process data $[8]$ is used here.

DTW was developed in the field of speech recognition [12] in order to provide a distance measure to quantify the similarity of two time series trajectories that is not too sensitive to small timing differences. This enables the audio signal of a spoken word to be classified according to its similarity to the audio signals in a labelled reference database. It has since become a widespread technique in all fields related to clustering or classifying time series data [13. As such, in most applications the synchronisation of the two trajectories is only a means to an end of obtaining the DTW distance. Conversely, in the batch process SPM field, it is the synchronisation step as a preprocessing tool, and not the DTW distance which has been the focus 8 .

The nearest neighbour principle has been previously applied to batch process 
data by He and Wang [14, who note that the approach is better suited to nonlinear and multimodal data than MPCA. However, the Euclidean distance was used which has been shown to be highly sensitive to timing differences [15] and online implementation of the method during an ongoing batch was not discussed.

A novel method for online statistical process monitoring of batch processes using DTW and kNN is presented. The method, which we refer to as DTWNN, compares an ongoing batch to all previous NOC batches using DTW. If the sum of the DTW distances to the $k$ nearest NOC batches suddenly increases, the batch is signalled out of control. This data-driven approach is conceptually simple, as an ongoing batch is compared directly to previously completed NOC batches, and their similarity assessed via DTW. In addition to the DTW distance monitoring statistic, the identification of the most similar historical batches provides the basis for a useful visualisation tool for process operators. The DTW-NN method makes no assumptions regarding the structure of NOC batch data, and is robust to the presence of clustering and non-linearity provided that enough representative data is available. The method is highly flexible as the reference database can be continually updated with newly completed NOC batches.

In the following sections DTW-NN is outlined in detail. It is applied to the extensive datasets from [16] of a simulated penicillin production batch process and performance is contrasted with that of a benchmark MPCA scheme.

\section{Methods}

Given a set of class-labelled points, $x_{i}$ for $i=1, \ldots, n$, the k-Nearest Neighbour $(\mathrm{kNN})$ classifier assigns a class to a new unlabelled point $x_{n+1}$ according to the majority class of the $k$ points closest in distance to $x_{n+1}$ [17]. The Euclidean distance is often the distance measure used to determine the k-nearest neighbours. When each $x_{i}$ is a time series, Wang et al. [15] have demonstrated the superiority of the DTW distance over the Euclidean distance for kNN classification for a wide range of applications and this distance measure will be discussed 
in detail in the following section.

It is the goal of fault detection of batch processes, to assign to a batch the class labels of either "NOC" or "Faulty". However, the available training data usually only consists of NOC batches and few if any faulty batches. Even if some faulty batches are available, if the classical kNN classification method is used on a new batch exhibiting an unseen type of fault, it is unlikely to be effective. Therefore, it is preferable that the fault detection method is built using only NOC data, in order to detect any deviations from NOC behaviour. He and Wang [14 adapted the $\mathrm{kNN}$ principle for fault detection by using the sum of distances to the $k$ nearest NOC batches. The distance between two batches was defined as the Euclidean distance between the unfolded batch data. The method was applied to complete batches of a semiconductor etch process. If the batch is faulty, this distance is expected to be large, where as if it is NOC there are likely to be similar past batches so the sum of distances will be small. The control limit for the sum of distances can be estimated from the distribution of its value for completed NOC batches.

We propose using DTW to identify the nearest neighbour NOC batches. DTW is well suited to the problem because batch processes often display variation in their rates of completion. A batch which is slightly slower than another batch but which otherwise has similar variable magnitudes and shapes would often result in a large Euclidean distance. In contrast, DTW is robust to small shifts, contractions and dilations in time and would therefore assign a small distance between the batches. Therefore DTW is a more appropriate measure of batch similarity. Furthermore, DTW can be used to estimate the similarity between a partially complete batch, and a complete batch whilst identifying the corresponding point in the complete batch which has been reached by the partial batch. This allows us to identify the nearest neighbour NOC batches, whilst a batch is in progress, in a way which is not sensitive to small differences in the speed of the ongoing batch. We focus on online monitoring which has greater relevance for those batch processes which take hours or days to complete and where there is time to take corrective action in response to any detected 
faults.

Existing methods involving DTW and batch process monitoring, rely on a single reference batch to which all other batches are aligned. The reference batch should be a typical NOC batch. A problem with this is that many processes do not have stable conditions. For example there may be seasonal variation, sensor drift, wear on equipment or changes in suppliers of raw materials which result in sudden or gradual changes in normal operating conditions. Such variation can lead to multi-modal distribution in the database of previous batches. The reference batch which was a typical batch at one time, may later be very atypical.

In the $\mathrm{kNN}$ approach, instead of using a single reference batch, we compare an ongoing batch to all past NOC batches. The basic outline of DTW-NN has two steps

1. At current time $t$ of an ongoing batch, perform open ended DTW to each past NOC batch, obtaining the DTW distance to each reference batch

2. Sum the k smallest DTW distances to obtain $D_{t}^{(k)}$. If $D_{t}^{(k)}-D_{t-1}^{(k)}$ exceeds the control limit, signal out of control

We found that it was better to monitor the rate of change in the sum of $k$ smallest DTW distances, rather than the sum of distances directly.

In the following sections we outline the DTW algorithm, and procedures for selecting DTW parameter values. Then the DTW-NN model is presented with procedures for selecting the number of neighbours to use, $k$, and calculating the control limits.

\subsection{Dynamic Time warping}

After its development in the field of speech recognition [12, DTW has become widely used in batch process monitoring [8, 18, 9, 19, 20] to process the batches such that they have the same number of observations, and so that events within each batch are synchronised. This is done by selecting a single reference batch, and aligning the other batches to this reference batch. Once same-length batches are obtained, bilinear monitoring methods like MPCA can be applied. 
Two multivariate sequences, $\mathbf{X}(m$ samples $\times J$ variables $)$ and $\mathbf{Y}(n$ samples $\times J$ variables) can be synchronised using DTW as follows. First the $n \times m$ local distance matrix $\mathbf{C}$ is constructed, where $C_{i, j}$ is the distance between the $i^{\text {th }}$ row of $\mathbf{X}$ and the $j^{\text {th }}$ row of $\mathbf{Y}$ (denoted by $\mathbf{x}_{i}$ and $\mathbf{y}_{j}$ respectively). For batch process data, usually the weighted squared Euclidean distance is used

$$
C_{i, j}=\left(\mathbf{x}_{i}-\mathbf{y}_{j}\right)^{T} \mathbf{W}\left(\mathbf{x}_{i}-\mathbf{y}_{j}\right)
$$

where $\mathbf{W}$ is a $J \times J$ diagonal matrix containing the variable weights, to be discussed in the following section.

DTW considers warping paths, $f$, through the local distance matrix $\mathbf{C}$ that represent mappings, between $\mathbf{X}$ and $\mathbf{Y}: f=f_{1}, f_{2}, \ldots, f_{T}$ where $f_{t}=(i, j)_{t}$. The warping path is usually subject to the following constraints:

- Boundary conditions: $f_{1}=(1,1)$ and $f_{T}=(m, n)$, i.e., the first elements of $\mathbf{X}$ and $\mathbf{Y}$ should be aligned to each other as well as their last elements

- Continuity: If $f_{t}=(i, j)$ then $f_{t+1}=(i+a, j+b)$ where $a, b \leq 1$, i.e., the warping path cannot skip cells in the local distance matrix

- Monotonicity: If $f_{t}=(i, j)$ then $f_{t+1}=(i+a, j+b)$ where $a, b \geq 0$, i.e., each pairing cannot go backwards in time in either $\mathbf{X}$ or $\mathbf{Y}$.

The accumulated distance between the two sequences under a warping, $f$, is given by

$$
D_{f}(\mathbf{X}, \mathbf{Y})=\frac{1}{n+m} \sum_{t=1}^{T} \omega_{t} C_{f_{t}}
$$

where $\omega_{t}=2$ if warping the function from $f_{t-1}$ to $f_{t}$ takes a diagonal step and $\omega_{t}=1$ otherwise. These step weights are used to compensate for warping paths of different lengths [12]. DTW identifies the warping function which minimises the accumulated distance $D_{f}$ and the corresponding value

$$
D=\min _{f}\left[D_{f}(\mathbf{X}, \mathbf{Y})\right]
$$

is the DTW distance between the two sequences. The optimal warping function is found using dynamic programming [12, so only a small subset of all possible $f$ need be considered in order to solve Eq. 3 . 
Additional constraints can be imposed on DTW to limit the amount of warping in the solution. For example, global constraints can be used to limit the maximum absolute time difference between the warped sequences and local constraints limit the slope of the warping function such that extreme contractions or expansions are not permitted.

To apply DTW online, whilst a batch is in progress, open-ended DTW can be used [8]. In this version of DTW the end-point boundary condition is relaxed so that $\mathbf{X}$ is aligned to a leading portion of $\mathbf{Y}$. Let $\mathbf{Y}^{(p)}(p \times J)$ denote the first $p$ samples of $\mathbf{Y}$, then open ended DTW solves

$$
D=\min _{f, p}\left[D_{f}\left(\mathbf{X}, \mathbf{Y}^{(p)}\right)\right]
$$

The resulting value of $p$ is the time-point in the reference batch which best corresponds to the current time in the ongoing batch.

\subsection{DTW Parameters}

The multivariate form of DTW should be used as for most batch processes several variables are present, and it is the aim that the DTW distance will be sensitive to disturbances in any variable. The variables may be scaled to remove the extreme differences in magnitude due to different engineering units. An appropriate scaling is to divide each variable by its mean range (found from calculating the range of each variable for each batch in the reference dataset, and taking the mean of these ranges) [8]. Note that DTW-NN is independent of the variable scaling used as the scaling effect is taken into account by the DTW variable weights, $\mathbf{W}$ in Eq. 1. Various approaches to calculating $\mathbf{W}$ were investigating whilst developing the DTW-NN method. Previous weighting methods [8, 18, were developed in the context of using DTW for data alignment, and so aim to up-weight variables which are better guides for alignment, and down-weight those which are not. However, applying these weighting methods in the DTW-NN monitoring approach lead to poorer performance, as they result in the DTW distance being dominated by only a few of the variables, hindering the detection of faults which do not affect these specific variables. 
Instead, a new weighting method which ensured that all of the variables contribute approximately equally to the DTW distance was found to lead to better performance.

To encourage a more equal contribution to the DTW distance, the variables that vary most from batch to batch should have a small weight in the local distance calculation of Eq. 1, and variables that have least batch to batch variation should have greater weight. In batch processes, the batch to batch variation of each variable may be dependent on time. Let $\sigma_{j, t}^{2}$ denote the variance across the $I$ reference batches of variable $j$ at time $t$. Then let

$$
\tilde{\sigma}_{j}^{2}=\operatorname{median}\left(\sigma_{j, t}^{2}\right)
$$

denote the median of the batch-to-batch variance of variable $j$ over the entire batch duration. The reason for taking the median batch-to-batch variance over the process duration, is to reduce the influence of extreme values, occurring for example in periods containing phase transitions. The median provides a more robust estimate of the average batch-to-batch variance over the entire process duration. Then we define $\mathbf{W}$ as the diagonal matrix where

$$
W_{j, j}=\frac{1}{\tilde{\sigma}_{j}^{2}}
$$

In addition, if batches appear to cluster into groups, the weights should be adjusted by calculating $\tilde{\sigma}_{j}^{2}$ for each cluster in the data and then averaging across clusters. This ensures that the weights are not distorted by betweencluster variation. When there is no prior knowledge regarding the presence of clusters, and which batches belong to which clusters, hierarchical clustering can be performed on the reference dataset, with the DTW distance in Eq. 3 as the between-batch distance measure. The DTW distance should be calculated using a subset of the $J$ variables which are most likely to be affected by clustering. The silhouette method from [21] may be used to judge whether clustering is present, and if so how many clusters. If clusters are detected, then $\tilde{\sigma}_{j}^{2}$ from Eq. 5 is calculated for each cluster, using only the batches in that cluster, and then the average of $\tilde{\sigma}_{j}^{2}$ is taken across clusters before applying Eq. 6. For further 
details regarding hierarchical clustering and the silhouette method we refer to 21.

A local constraint should be used in order to avoid singularities in the DTW alignments. Without a local constraint, the alignment is free to compress long sections of the batch to an instant, or "freeze" one time sample for a prolonged duration, in order to synchronise two batches. It is expected that batches run faster or slower than each other, but not that they stop completely or suddenly jump forward in time. The local constraints suggested in [12] are parametrised by the value $P$, which represents the number of diagonal steps the warping function must take for each horizontal or vertical step. A method for selecting the value of $P$ may be found in [20] and is applied in this work.

Finally, a global constraint may be applied to shorten computational time as it reduces the need to calculate every pairwise local distance. We use the simplest type of global constraint known as a band constraint. This limits alignment of point $t$ in an ongoing batch to some point in the interval $[t-w, t+w]$ in the reference dataset batch. For $w$ we used the range of the durations of the reference batches.

\subsection{DTW-NN model specification}

Suppose a dataset of $I$ NOC batches is available. Then, the DTW-NN model is specified as follows.

First, the $I$ batches are used to determine the DTW parameters $(\mathbf{W}$, local constraint and global constraint) as described in the previous section. Then the first batch is treated as an ongoing batch $\mathbf{X}$, and the remaining $I-1$ batches as the reference dataset. For each sample time $t$ in $\mathbf{X}$, open ended DTW is performed between the first $t$ rows of $\mathbf{X}$ and every other batch in the reference dataset and the resulting DTW distances are saved. At each time point the smallest $k$ distances are summed to obtain the quantity $D_{t}^{(k)}$, which is a measure of the similarity between the entire variable trajectories of the ongoing batch up to time $t$, and $k$ nearest NOC batches. If a fault occurs at time $t+1$ which influences one or more of the variable trajectories, $D_{t+1}^{(k)}$ may not 
necessarily immediately jump to a large value, because it takes into account the similarity over the entire batch duration so far, which up until time $t+1$ was entirely normal. However, we expect to observe an increase in $D_{t+1}^{(k)}$ relative to its previous value. Therefore, the change in nearest neighbour distance is calculated as

$$
\begin{aligned}
& \dot{D}_{1}^{(k)}=D_{1}^{(k)} \\
& \dot{D}_{t}^{(k)}=D_{t}^{(k)}-D_{t-1}^{(k)} \text { for } t=2, \ldots, N
\end{aligned}
$$

At the end of the batch, let $D^{(k)}$ and $\dot{D}^{(k)}$ be vectors where the $t^{\text {th }}$ elements are $D_{t}^{(k)}$ and $\dot{D}_{t}^{(k)}$ respectively. This procedure is repeated for the remaining batches, such that each in turn is treated as the ongoing batch and $D^{(k)}$ and $\dot{D}^{(k)}$ vectors are obtained for each batch in the reference database.

The $\dot{D}^{(k)}$ vectors are arranged in a $I \times N$ matrix, $\dot{\mathbf{D}}$, where the $i^{\text {th }}$ row of $\dot{\mathbf{D}}$ is $\dot{D}^{(k)}$ for the $i^{t h}$ batch, and $N$ is the length of the longest reference batch. The duration of batches can vary so there may be missing values in the final columns of $\dot{\mathbf{D}}$. As $\dot{\mathbf{D}}$ contains the rates of change of the $\mathrm{kNN}$ distance throughout each NOC batch in the reference dataset, it can be used to calculate $100(1-\alpha) \%$ control limits. The rate of change in $\mathrm{kNN}$ distance is time dependent and is typically larger at the start of a batch. Therefore, time dependent control limits are calculated. The theoretical distribution $\dot{D}_{t}$ is not known so the quantile is calculated empirically for each column of $\dot{\mathbf{D}}$. A Gaussian kernel is used to smooth the empirical probability density of each column and obtain more reliable estimates of the extreme quantiles used for control limits (e.g. $\alpha=0.01$ ). For the final columns of $\dot{\mathbf{D}}$ that contain missing values, the control limits can still be calculated using only the non-missing values. The accuracy of the control limits will decrease as the number of missing values increases, but so too will the expected number of future batches that will still be in progress for those time-points.

The number of nearest neighbours, $k$, influences the smoothness of $D^{(k)}$ so to increase stability $k$ should be greater than one. Conversely, if $k$ is too large, $D^{(k)}$ becomes less sensitive to local structures in the reference dataset and also 
$k$ needs to be less than the size of any known batch clusters. We devised the following unsupervised method for selecting $k$.

Consider the first nearest neighbour of a batch in the reference dataset. The first nearest neighbour is likely to change throughout the batch duration. Let $\kappa_{i}$ represent the number of unique batches observed within the first nearest neighbour to batch $i$ throughout the duration of batch $i$. Calculate $\kappa_{i}$ for $i=1, \ldots, I$. Let $k=\operatorname{mode}\left(\kappa_{i}\right)$.

The motivation for this approach is that we often observed the nearest neighbours to change places with each other over the course of a batch. For example, for time 1 to 50 batch 11 may be the first nearest neighbour and batch 32 the second nearest neighbour, then at time 51, batch 32 is the first nearest neighbour and batch 11 the second nearest neighbour. By letting $k=\operatorname{mode}\left(\kappa_{i}\right)$ it is hypothesised that for the most part the $k$ nearest neighbours will consist of the same $k$ batches throughout the duration of the batch, and this will minimise instability caused by changes in members of the set of $k$ nearest neighbours. It should be noted that the value of $k$ obtained using the above method will generally increase with $I$, the number of batches in the reference set. This is reasonable, because a bigger reference dataset will contain a greater number of batches that are similar to a new batch, so the monitoring statistic should take into account the distance to more nearest neighbours.

\subsection{Monitoring a new batch with $D T W-N N$}

To monitor a new batch using DTW-NN, let $\mathbf{X}$ be a new batch for which data has been collected up until the $t^{\text {th }}$ sample. Then the DTW distance is calculated between $\mathbf{X}$ and each batch in the reference dataset. The smallest $k$ distances are summed to obtain $D_{t}^{(k)}$ and the change in the nearest neighbour distance is calculated using (7) to obtain $\dot{D}_{t}^{(k)}$. Finally, an out of control signal is generated if $\dot{D}_{t}^{(k)}$ exceeds the control limit for time $t$.

In addition to the $\mathrm{kNN}$ distance statistic, this model provides valuable information for online data visualisation by identifying the $k$ nearest neighbours at each time-point of an ongoing batch. The variable trajectories of the $k$ nearest 
neighbour batches can be plotted together with the partially complete trajectories of the ongoing batch to provide insight to operators on how the ongoing batch compares to historical data.

\section{5. $M P C A$}

To assess the performance of the proposed method, we compare it to the MPCA method introduced by [1, 22], where the data is aligned with DTW following [8]. This method is based on fitting a PCA model to the unfolded NOC reference dataset as follows.

First the $I$ reference batches are scaled by dividing each variable by its mean range over the $I$ batches. Next, a reference batch is selected, to which the remaining batches are aligned using DTW such that all batches then have the same number of observations as the reference batch, $N$. The variable weights, $\mathbf{W}$ used in this alignment are here determined using the method in [18] which was developed to give greater weights to those variables which are most indicative of process time. To avoid extreme warpings, a local constraint is selected according to [20], and a band global constraint of width equal to the range of the batch durations is used.

Let $\underline{\mathbf{Z}}(I \times J \times N)$ be a three-way data matrix formed by the aligned NOC reference data set (where $N$ is the length of the reference batch). This matrix is unfolded batch-wise to form $\mathbf{Z}(I \times J N)$. The columns of $\mathbf{Z}$ are mean centred and scaled to unit variance and a PCA model is fitted, where $L$ components are retained. The loading vectors from this model, as well as the column means and standard deviations, are saved for the online implementation.

For online monitoring of an ongoing batch at time $t$, the first step is to align the existing section of ongoing batch to the reference batch using openended DTW. The aligned data is then unfolded, centered and scaled using the previously saved parameters. As the ongoing batch is only partially complete, it is necessary to estimate the future observations in some way so the the scores can be calculated using the PCA model. We use the projection to the model plane approach from [22]. Then the PCA model is applied to obtain the current scores 
and the monitoring statistics of Hotelling's $T^{2}$ and $Q$ (the squared prediction error of the model at the current time point). Following [23, 24, we used a time dependent mean and covariance to calculate $T^{2}$ which is determined from the reference dataset using a leave one out procedure. The $100(1-\alpha) \%$ control limit for Hotelling's $T^{2}$ is given by

$$
T_{\alpha}^{2}=\frac{L\left(I^{2}-1\right)}{I(I-L)} F_{L, I-L}(\alpha)
$$

where $F_{L, I-L}(\alpha)$ is the $(1-\alpha)$ quantile of the $F$ distribution with $L$ and $I-L$ degrees of freedom.

The control limits for $Q$ were calculated using the standard approach based on the $g \chi_{h}^{2}$ distribution with $g$ and $h$ estimated for each time point from the reference dataset using the method of moments [22].

\section{Results and Discussion}

\subsection{The Pensim datasets}

To evaluate the proposed method, the four simulated datasets provided by [16] were used. These datasets, which extend the Pensim model of [25], represent data from batch production of penicillin. The simulation was designed to be as realistic as possible, for example, with regards to sensor measurement noise, batch to batch variability of initial conditions and automatic control mechanisms. Each of the four datasets represents different assumptions about the production process which are briefly summarised as follows

- Dataset 1: "Base". Initial conditions independently sampled from normal distributions

- Dataset 2: "Skewed". Some of the initial conditions sampled from uniform distribution, and non-linear dependence introduced between some initial conditions

- Dataset 3: "Tail". One of the parameters of the Pensim model is drawn from a $\chi^{2}$ distribution. Initial conditions are generated the same way as for dataset 2 . 
- Dataset 4: One of two types of micro-organisms is used for each batch. Initial conditions are generated the same way as for dataset 2 .

Each dataset consists of 400 NOC batches and several thousand faulty batches. The faulty batches consist of 15 different fault types, each with a range of different fault magnitudes. Additionally, the faults start in one of four different time intervals. The original dataset contains 50 repetitions of each type/magnitude/onset-time-interval combination for a total of 22,200 faulty batches. To save computational time, we reduced this to 5 repetitions per combination by random sampling, resulting in a total of 2220 faulty batches per dataset. For further details on the simulation of the datasets we refer to the original work [16].

Table 1: Variables measured online in Pensim process and abbreviations used in this paper

\begin{tabular}{ll}
\hline Variable & Abbreviation \\
\hline Time $(\mathrm{h})$ & Time \\
Volume $\left(\mathrm{m}^{3}\right)$ & Volume \\
Dissolved Oxygen concentration $(\mathrm{mg} / \mathrm{L})$ & $\mathrm{O} 2$ \\
Dissolved $\mathrm{CO}_{2}$ concentration $(\mathrm{mg} / \mathrm{L})$ & $\mathrm{CO} 2$ \\
Reactor temperature $(\mathrm{K})$ & $\mathrm{RctrTemp}$ \\
$\mathrm{pH}(-)$ & $\mathrm{pH}$ \\
Feed rate $(\mathrm{L} / \mathrm{h})$ & FeedRate \\
Feed temperature $(\mathrm{K})$ & FeedTemp \\
Agitator power $(\mathrm{W})$ & Agitator \\
Cooling water flow rate $(\mathrm{L} / \mathrm{h})$ & WaterRate \\
Base Flow Rate $(\mathrm{L} / \mathrm{h})$ & BaseFlow \\
Cumulative base flow $(\mathrm{mL})$ & BaseQty \\
Cumulative acid flow $(\mathrm{mL})$ & AcidQty \\
\hline
\end{tabular}

The 12 variables listed in table 1 are measured online throughout each batch and will be used for monitoring. In the original dataset the variables are measured every 0.2 hours resulting in around 2300 samples per batch. To reduce 
In the following sections we present detailed results of applying DTW-NN and MPCA to dataset 4 which displays the multi-modal features that we expect DTW-NN to be especially suited to. In section 3.6 we summarise the findings of applying the methods to all four datasets.
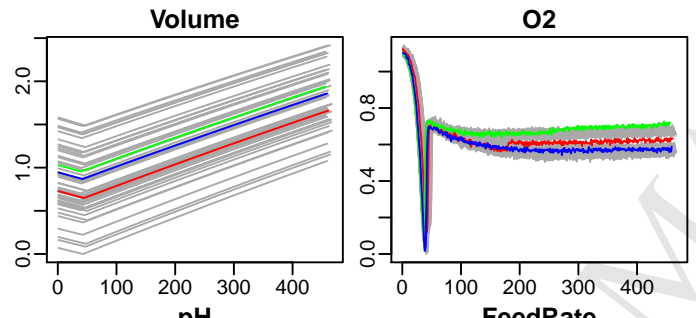

$\mathrm{pH}$
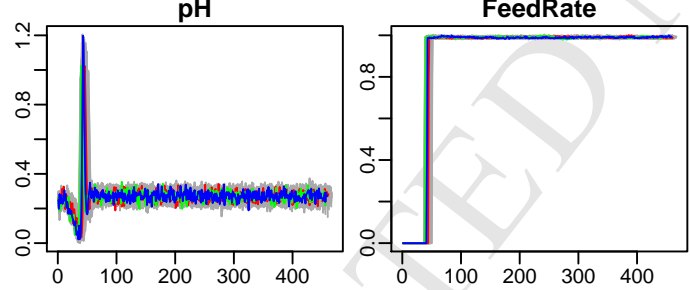

WaterRate
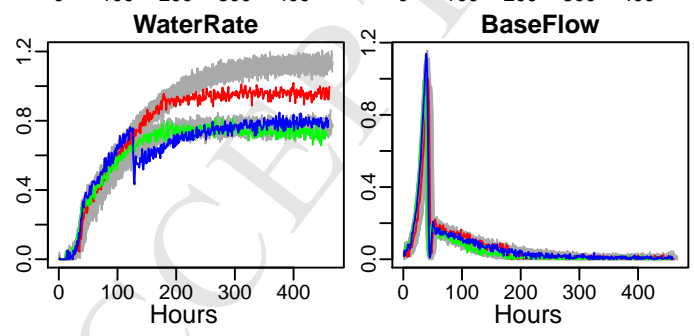

$\mathrm{CO} 2$

FeedTemp
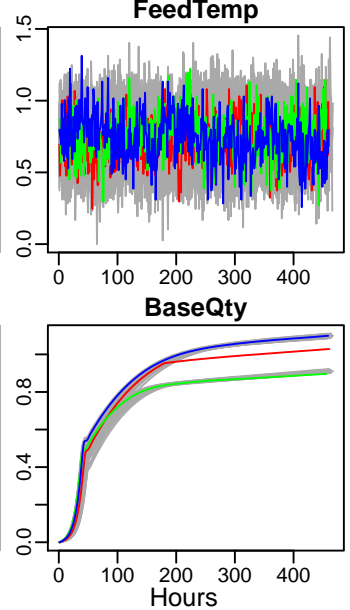

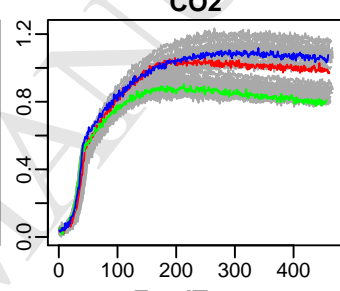

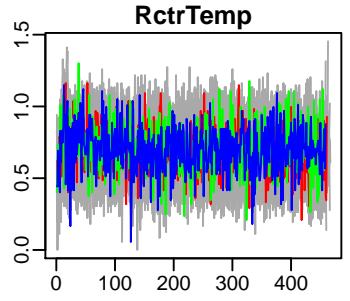

Agitator
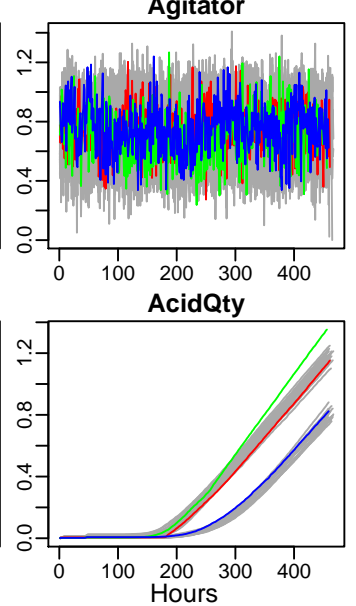

Figure 1: Variable trajectories of the 50 NOC batches randomly selected as the reference dataset (grey), and 3 faulty batches (red, blue, green), after scaling by mean range. 
variables (O2, CO2, WaterRate, BaseQty, AcidQty) show multi-modality as a result of the two different strains used for different batches. In a real scenario, this type of variation may be observed due to using different suppliers, or different batches of raw material from the same supplier. Monitoring performance will be summarised for all 2220 faulty batches, but three faulty batches were selected for more detailed illustration and are also shown in Fig. 1. These are the red batch (fault type 1: $-10 \%$ change in feed substrate concentration at time $178 \mathrm{hrs}$ ), the blue batch (type $2:-10 \%$ in coolant temperature at time $125 \mathrm{hrs}$ ) and the green batch (type 15: simulated contamination at time $254 \mathrm{hrs}$ ).

\subsection{DTW parameter tuning}

As there is clearly clustering in the data, it was appropriate to apply hierarchical clustering before calculating the variable weights. The dataset contained information on which strain was used for each batch, so the correct clustering is known, but we chose to try and identify the clustering empirically without using this information. To perform the clustering, the DTW distance was calculated between every pair of batches in the reference database, based on the variables BaseQty, WaterRate and O2. The silhouette method clearly identified two clusters as most appropriate, so the hierarchy was cut at two clusters. Comparing to the strain labels showed that this approach had correctly classified all the batches. It must be noted that in this dataset the clustering of batches, and the variables most indicative of clustering, are quite easily visible in Fig. 1. Other cases may pose a greater challenge to successful clustering. In such cases, prior knowledge of possible clusters due to, for example, different suppliers of raw materials, would be useful.

Next, for each variable, and for each cluster, the median over time of batch to batch variance was calculated (Eq. 5). Then, the average of the variance estimates from the two clusters was taken before calculating the final weights (Eq. 6). The resulting weights are shown in Fig. 2.

Next, using the method in [20], a local constraint of $P=1$ was selected. This constraint enforces one diagonal step for each vertical or horizontal step 


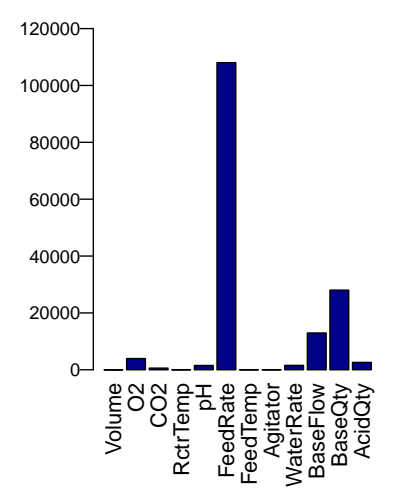

Figure 2: Variable weights for DTW

taken by the warping function.

Finally, the range of the durations of the 50 reference batches was 12 . Hence the shortest batch was 12 hours shorter than the longest batch. If a new batch is in NOC we will not expect it to be more than 12 hours faster or slower than any of the reference batches at any point, and therefore a band global constraint of \pm 12 samples was selected for DTW.

\subsection{DTW-NN model specification}

For each batch in the reference database, online DTW was performed to the other 49 reference batches, and the nearest neighbours to the batch were identified throughout its duration. To select $k$, the number of unique batches identified as the first nearest neighbour was examined. For example, Fig. 3 shows the online DTW distance throughout batch 19 to every other reference batch. The nearest neighbour batch to batch 19 changes several times but from $t=70$ onwards remains constant at reference batch 28 . In total, 5 different reference batches were identified as the nearest neighbour to batch 19 throughout its duration. The distribution of the number of unique batches appearing as the nearest neighbour throughout every reference batch is shown in Fig. 4 , and we selected $k=8$ from the mode of this distribution.

Next, the sum of the 8 nearest neighbour distances, $D^{(8)}$ was calculated 

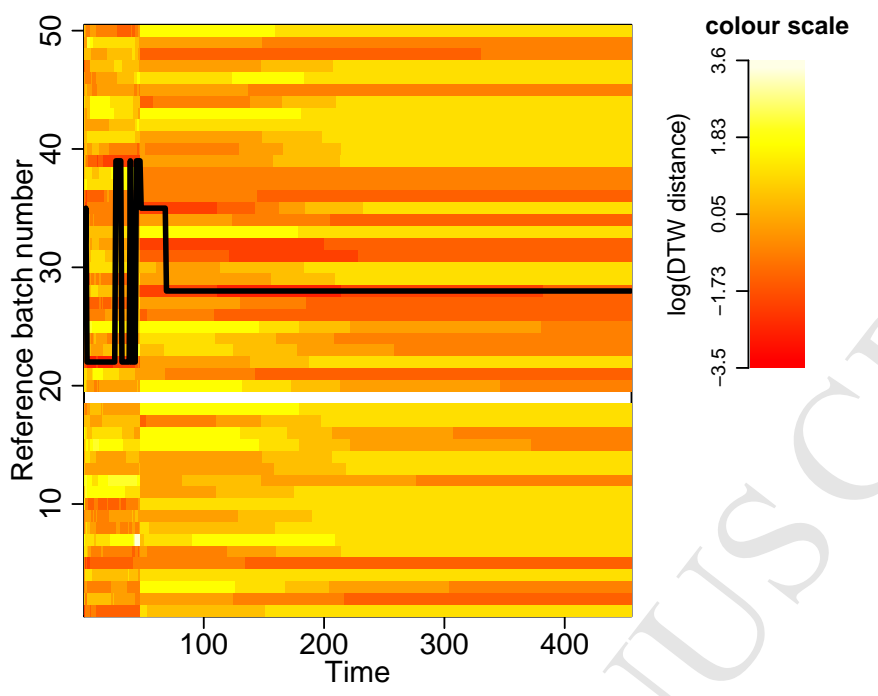

Figure 3: Heatmap of the online DTW distance between batch 19 and the other 49 batches in the reference dataset, throughout the duration of batch 19. The black line indicates the nearest neighbour to batch 19 at each time point.

throughout each batch. The value is shown for the 50 reference batches in Fig. 5. as well as for the three faulty batches. This illustrates the motivation for monitoring the change in $D^{(8)}$ rather than $D^{(8)}$ itself, as after each fault a clear increase in $D^{(8)}$ is observed even though the value of $D^{(8)}$ is not necessarily large compared to the values observed for the NOC reference batches. Therefore, $\dot{D}^{(8)}$ was calculated. In order to compare performance over the same time periods as MPCA, we truncated $\dot{D}^{(8)}$ to the length of the shortest batch (455). To aid visualisation, $\dot{D}^{(8)}$ was mean centered and scaled to unit variance at each time point using sample statistics from the 50 reference batches. An $\alpha=0.01$ control limit was calculated for each time point by smoothing the sample probability distribution of $\dot{D}^{(8)}$ from the 50 reference batches with a Gaussian kernel. This confidence limit, and $\dot{D}^{(8)}$ for the 50 reference and red, blue and green batches is shown in Fig. 5 . Before evaluating performance across all 2220 faulty batches, results regarding specification of the MPCA benchmark model will be presented. 


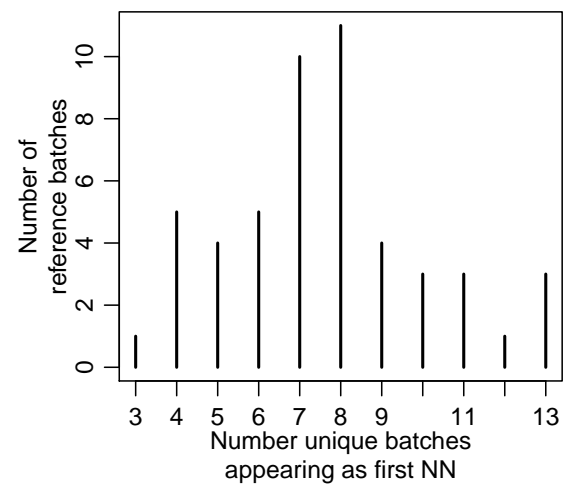

Figure 4: Distribution of the number of unique batches appearing as the first nearest neighbour of each reference batch

\subsection{MPCA model specification}

The MPCA monitoring model was built as described in section 2.5. For DTW alignment of the data, the reference batch was selected as the batch closest to median duration, and a local constraint of $P=1$ was used following [20] and variable weights were determined using [18. The reference dataset of 50 batches was aligned and unfolded such that each variable at reference times 1 to 455 was placed side by side. This $50 \times 5460$ matrix was centered and scaled and a PCA model was fit. Based on the scree plot of the eigenvalues (Fig. 6), 5 principal components were retained in the model. The scores were predicted online throughout all batches, with projection to the model plane as the infilling method. This included online alignment to the reference batch using open-ended DTW. Online Hotelling's $T^{2}$ and $Q$ statistics were calculated from the scores, as well as their $\alpha=0.01$ control limits.

\subsection{Performance evaluation}

Three key performance indicators (KPI) were adopted for the evaluation of monitoring performance:

- FAR (False Alarm Rate) = proportion of samples exceeding the control limits whilst the batch is in a NOC state. 


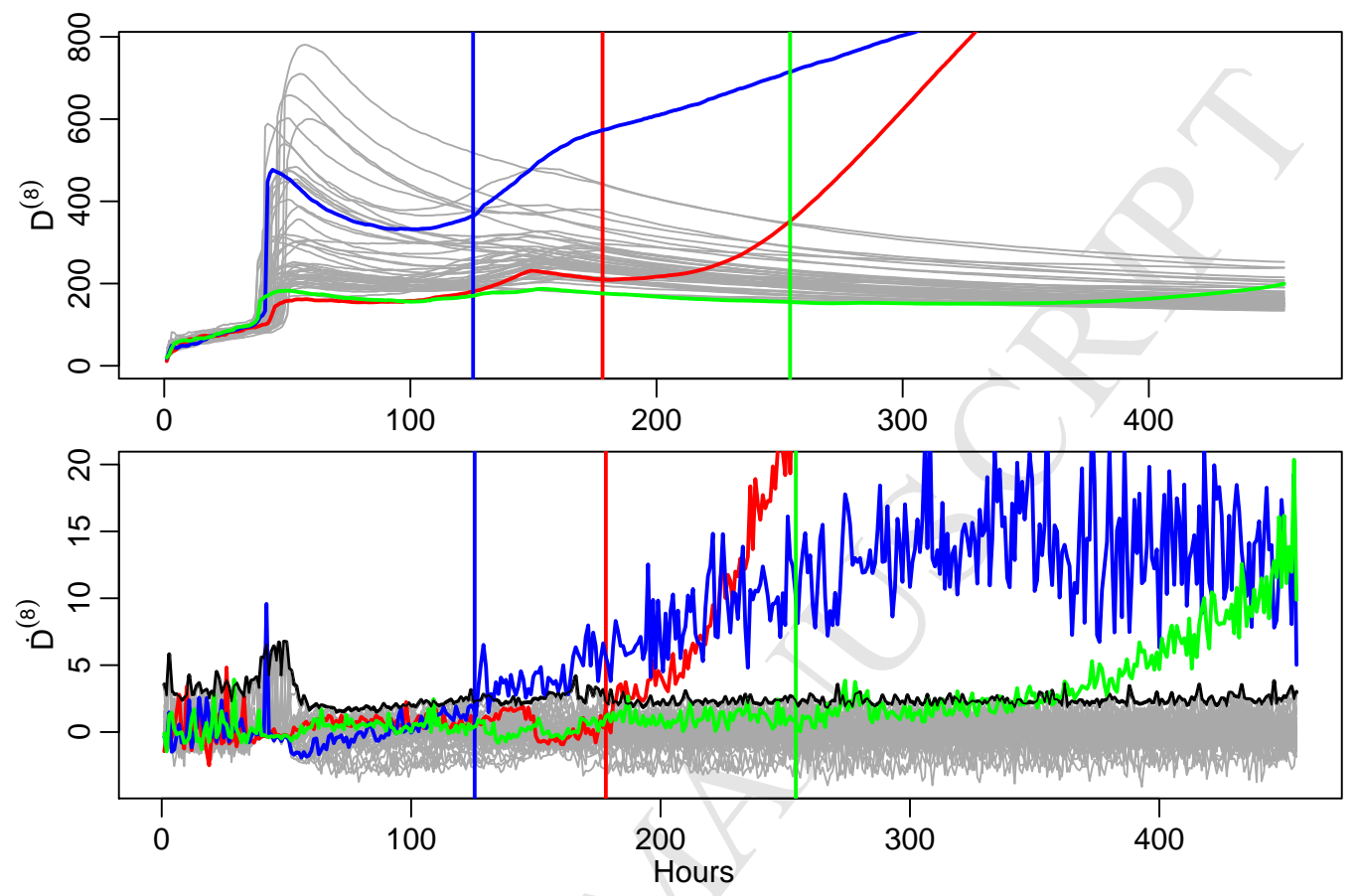

Figure 5: Sum of 8 nearest neighbour distances over time (top) and the slope over time (bottom) for the 50 reference batches (grey) and the red, blue and green faulty batches. The vertical lines indicate the fault onset times. The black line is the control limit.

- TDR (True Detection Rate) = proportion of samples exceeding the control limits whilst a batch is in a faulty state.

- $\operatorname{TTS}_{a}($ Time To Signal $)=$ the number of samples observed before $a$ consecutive samples exceeded the control limits, since the onset of a fault. If $a$ consecutive samples never exceed the limits we define TTS for that batch as $\infty$.

These indicators were calculated for each batch individually. The batches were defined as in a faulty state for all observations after the fault onset time, and as in a NOC state for all observations prior to the fault onset. The 400 NOC batches were in a NOC state for their entirety.

Table 2 contains KPI summary statistics for the DTW-NN and MPCA meth- 


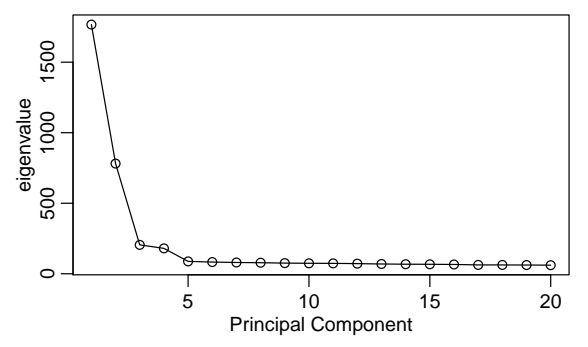

Figure 6: Scree plot of first 20 eigenvalues of the MPCA model

Table 2: Mean FAR of the 350 NOC batches not in the reference dataset, and mean TDR, median $\mathrm{TTS}_{1}$, median $\mathrm{TTS}_{3}$ of the 2220 faulty batches

\begin{tabular}{rrrrr}
\hline & FAR & TDR & TTS $_{1}$ & TTS $_{3}$ \\
\hline DTW_NN & 0.0119 & 0.5457 & 8.0 & 32.0 \\
MPCA_Q & 0.0071 & 0.3403 & 16.0 & $>362.0$ \\
MPCA_T & 0.0009 & 0.1613 & $>445.0$ & $>344.0$ \\
MPCA_Q-or-T & 0.0079 & 0.3517 & 16.0 & $>362.0$ \\
\hline
\end{tabular}

ods. In evaluating the two methods we must account for the fact that the MPCA model consists of two monitoring statistics, whilst the DTW-NN model has a single monitoring statistic. Table 2 shows that the mean TDR for the combined $Q-\mathrm{OR}-T^{2}$ alarm is only marginally better than TDR for $Q$ alone. In addition, in 2164 of the 2220 faulty batches, TDR for $Q$ exceeded TDR for $T^{2}$. Therefore, the Hotelling's $T^{2}$ of the MPCA model is made almost entirely redundant by the $Q$ statistic. For these reasons, we hereafter focus on comparing the performance of DTW-NN to the MPCA $Q$ chart.

To make a fair comparison between two monitoring statistics, it is important that the FAR is similar for both of them. Table, 2 shows that mean FAR of DTW-NN is close to that of MPCA- $Q$, and they are both close to the target value of $\alpha=0.01$. Their distributions also do not differ greatly (Fig. 7). Despite this, mean TDR is much higher for DTW-NN than for MPCA- $Q$, so we conclude that DTW-NN is the superior method for this dataset. A mean TDR of $54 \%$ for DTW-NN may not appear large, but it is important to note that the dataset 

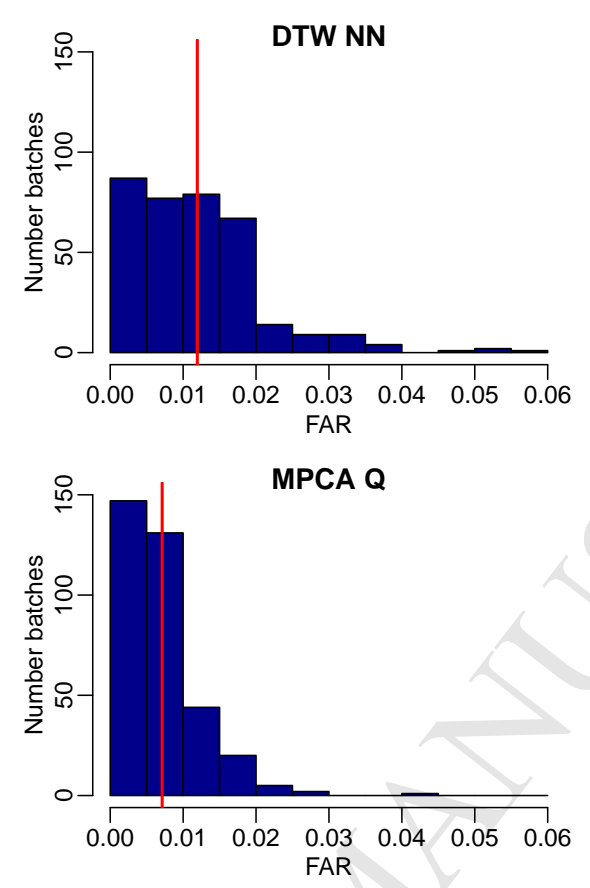

Figure 7: Distribution of FAR for the two methods, with mean value shown by the vertical lines

contains a range of fault magnitudes, many of which are very mild.

In Fig. 8 TDR of the two methods is plotted for all 2220 faulty batches, and shows that DTW-NN performance is as good as or better than MPCA- $Q$ for 13 of the 15 fault types. Only for fault type 3 and fault type 9 (Agitator power drop and Non-functioning $\mathrm{pH}$ control respectively) does MPCA-Q appear to perform systematically better than DTW-NN. Fig. 9 shows the online monitoring statistics for the three example batches. For these batches, DTW-NN clearly signals a deviation from the behaviour of historical batches, whilst MPCA- $Q$ does not detect anything unusual.

\footnotetext{
${ }^{1}$ The MPCA-Q TDR plot for Fault 6 reproduces closely the results presented in Fig. 14(d) of [16] with minor differences likely due to choice of MPCA infilling method, control limits, and our use of 5 batch repetitions rather than 50 .
} 

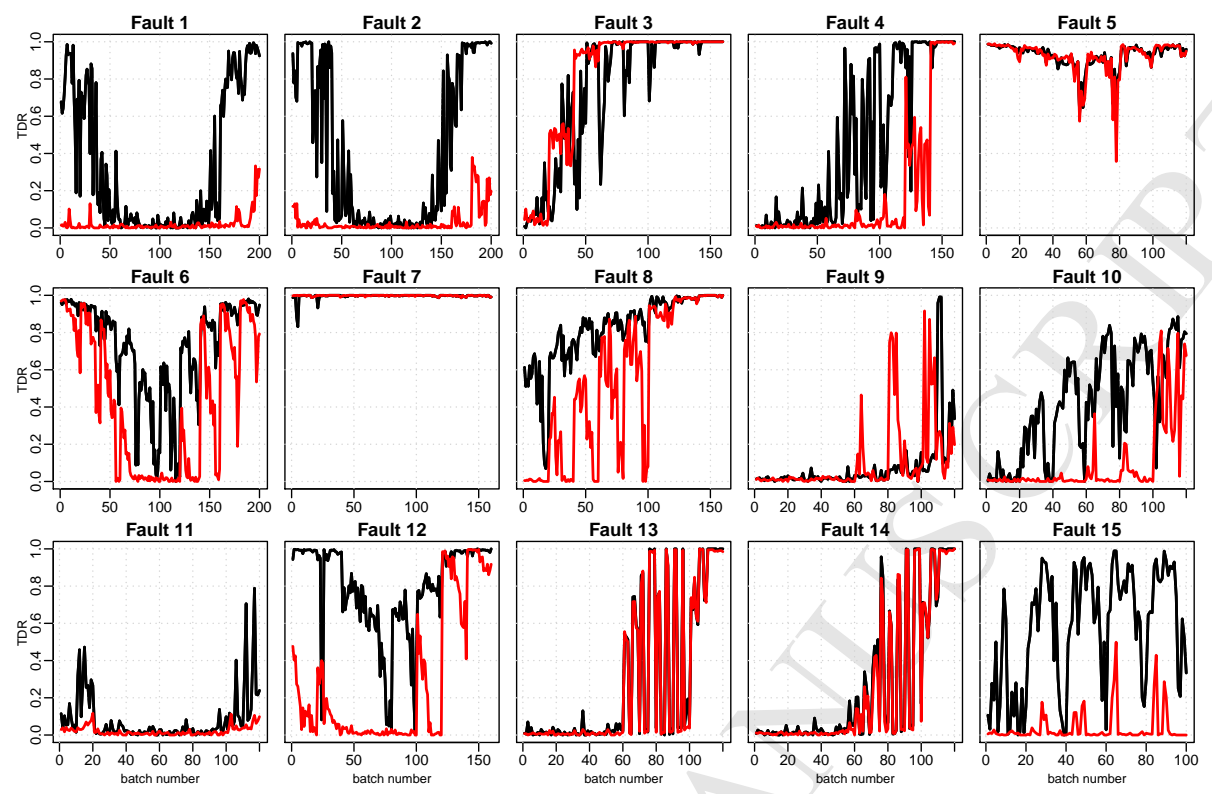

Figure 8: TDR of DTW-NN (black) and MPCA-Q (red) for all faulty batches ${ }^{1}$

The speed of detection for DTW-NN is also faster than for MCPA- $Q$ with median $\mathrm{TTS}_{1}$ values of 8 and 16 respectively (Table 2). For $\mathrm{TTS}_{3}$, DTW-NN has a median value of 16 whilst most the median for MPCA- $Q$ is over 344 .

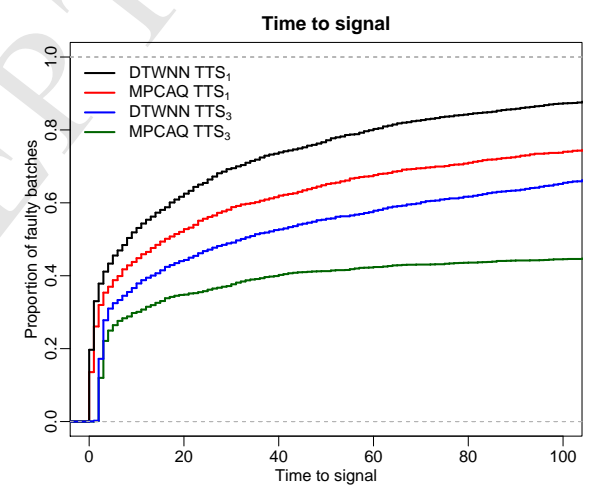

Figure 10: Observed cumulative distribution of $\mathrm{TTS}_{1}$ and $\mathrm{TTS}_{3}$ 
DTW NN
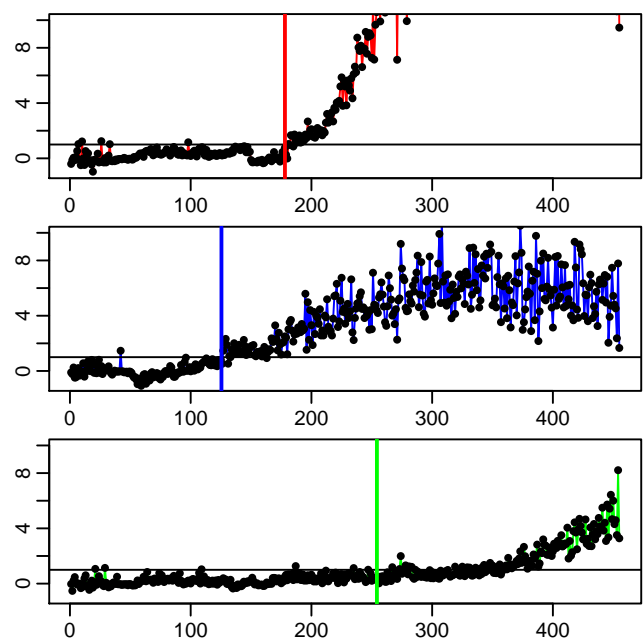

MPCA Q
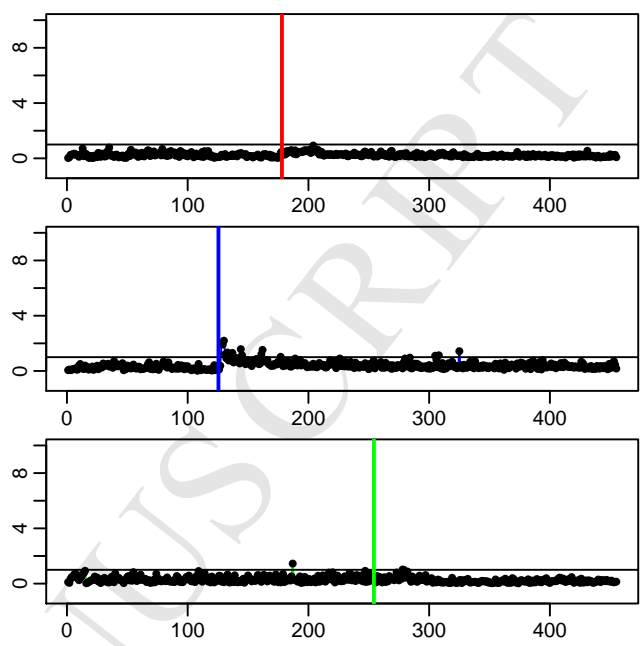

Figure 9: Monitoring results for the red (top), blue (center) and green (bottom) faulty batches with DTW-NN (left) and MPCA-Q (right). For easier comparison, the statistics have been divided by their control limit at each time point

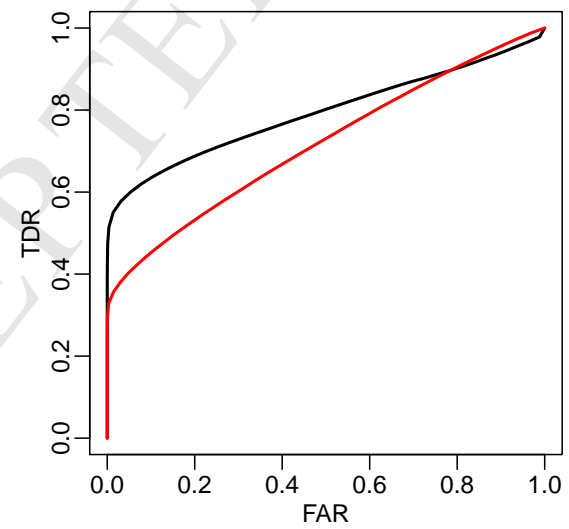

Figure 11: ROC Curve for DTW-NN (black) and MPCA- $Q$ (red)

As discussed in, for example, 23, a useful tool for comparing monitoring methods independently of a specific FDR is the ROC curve. We constructed 
the ROC curves in Fig. 11 by calculating control limits for many values of $\alpha$ in the range $[0,1]$ and for each value calculating the mean TDR and FAR over all faulty batches. The ROC curve confirms that the better performance of DTW-NN on this dataset cannot be attributed to chance differences in control limits.

It was of interest to examine the effect of number of neighbours, $k$, on performance and check the suitability of the proposed method for selecting $k$ from section 2.3 . The preceding analysis was repeated for different $k$ and the results are shown in Fig. 12. In fact, the selected value of $k=8$ had the second-highest TDR value, whilst a value of $k=7$ results in the highest TDR, though the difference in TDR is marginal (less than 0.001), Fig. 12 shows that TDR is not very sensitive to choice of $k$ in the range $k=2$ to 10 . TDR for $k=1$ is noticeably lower than $k=2$. This may be because when a fault occurs, there is greater likelihood of finding a single past NOC batch that somewhat resembles the faulty batch, than there is of finding two NOC batches that resemble the faulty batch. Mean TDR drops to a much lower value for $k>23$, as then $\mathbf{D}^{(k)}$ is forced to include distances to NOC batches in a different cluster than the ongoing batch.

The effect of the size of the reference dataset, $I$ on the selected value of $k$ using the proposed decision rule was examined by supposing the reference dataset consisted of $I=1,2, \ldots, 50$ reference batches and applying the rule in each case (Fig. 13). It appears that the value of $k$ selected using the decision rule can be approximated by $\sqrt{I}$, and this provides a quicker method for specifying $k$, which would be useful if new batches are continuously added to the reference dataset in a production setting.

\subsection{Other Pensim datasets}

The results of applying the methods to datasets 1,2 and 3 will now be presented together with the dataset 4 results. The same procedures were followed as previously described for dataset 4 . As there is no indication of clustering of batches in datasets 1 to 3 , the DTW weights were calculated using all 50 refer- 

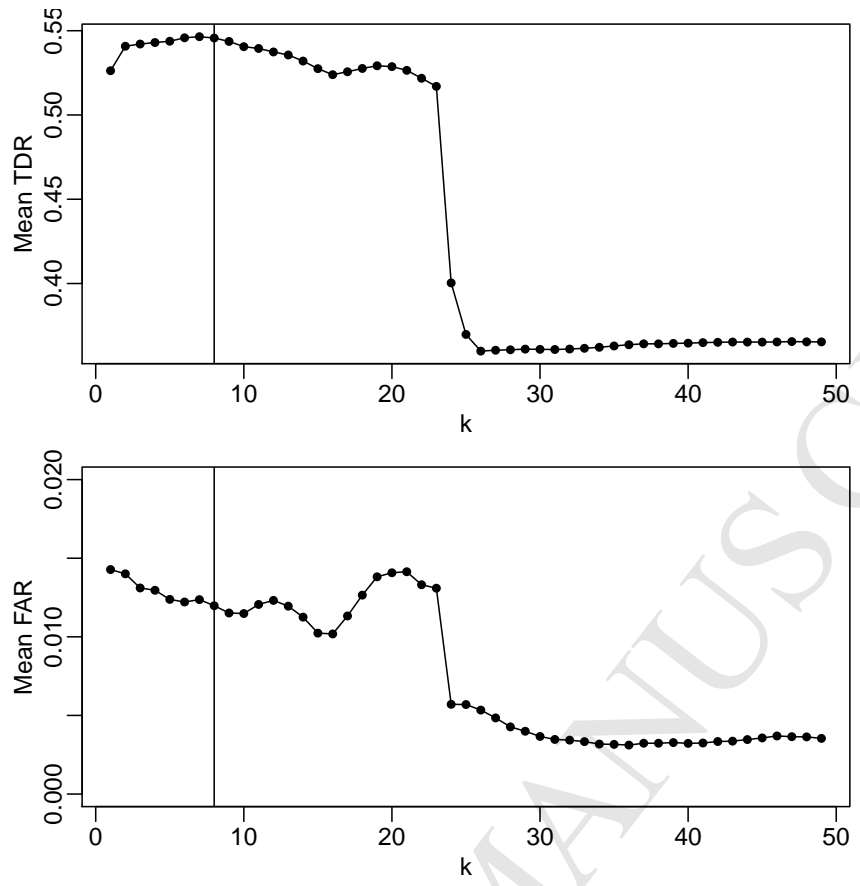

Figure 12: Mean TDR (top) and mean FAR (bottom) for $k=1, \ldots, 49$. The vertical line shows the value used, $k=8$.

ence batches, without the cluster identification step. The DTW-NN parameter $k$ was selected in the same way for each dataset. The number of components retained in the MPCA model was 5 for each dataset, and the MPCA model was trained and applied as described previously.

The performance of both methods' monitoring statistics for all four datasets is summarised in Fig. 14 and we highlight the following observations

- MPCA-Q shows marginally higher TDR than DTW-NN on dataset 3, but substantially lower TDR on dataset 4

- DTW-NN shows a more stable TDR across the four datasets (ranging from 0.51 to 0.61 ) than MPCA- $Q$ (ranging from 0.34 to 0.58 )

- For all the datasets, monitoring both $Q$ and $T^{2}$ from MPCA, results in only a minor improvement in TDR compared to only monitoring $Q$, and a substantially higher FAR (for datasets 1 to 3 in particular). 


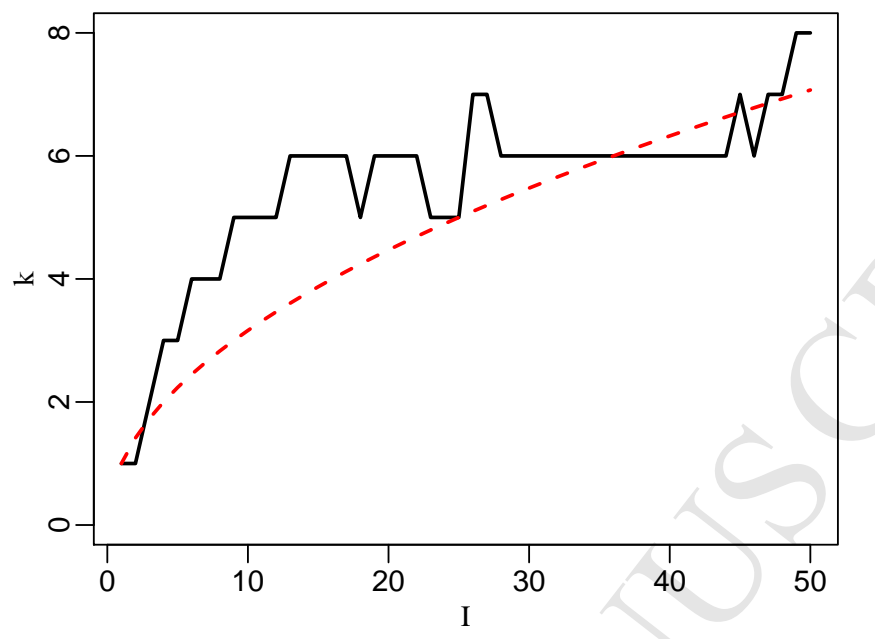

Figure 13: The value of $k$ (solid black line) selected for difference sizes of reference dataset, I. For comparison $\sqrt{I}$ (dashed red line) is also shown

- For both MPCA- $Q$ and DTW-NN, the observed mean FAR deviates from the target value of $\alpha=0.01$ for different datasets. Mean FAR varies from 0.0071 to 0.0191 for MPCA- $Q$ and from 0.0045 to 0.0153 for DTW-NN.

The validity of the proposed rule for selecting $k$ was also examined for datasets 1 to 3 , by comparing the selected value $\left(k_{\text {selected }}\right)$ to the value which resulted in the greatest mean TDR $\left(k_{\max T D R}\right)$ as shown in Table 3 . For datasets 1 to 3 , mean TDR for $k_{\text {selected }}$ and $k_{\text {maxTDR }}$ differs by at most 0.0144 (for dataset 3 ), supporting the view that mean TDR is not highly sensitive to $k$, and that the selection rule is a reasonable approach for selecting $k$ that gives a close to optimal TDR. 


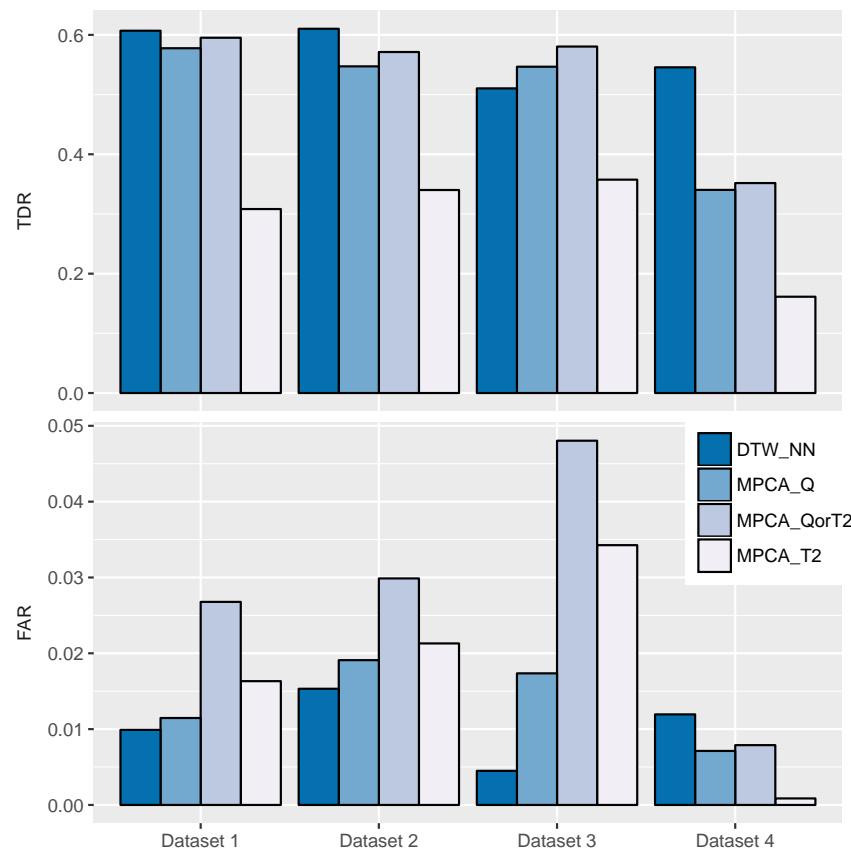

Figure 14: Mean TDR (top) and FAR (bottom) for MPCA and DTW-NN on all four sets.

Table 3: Selected $k$ values, and maximum TDR $k$ values for each dataset

\begin{tabular}{lll}
\hline Dataset & $k_{\text {selected }}$ & $k_{\text {maxTDR }}$ \\
& $($ mean TDR $)$ & $($ mean TDR) \\
\hline 1 & $8(0.6070)$ & $7(0.6104)$ \\
2 & $6(0.6102)$ & $11(0.6133)$ \\
3 & $6(0.5103)$ & $2(0.5248)$ \\
4 & $8(0.5457)$ & $7(0.5465)$ \\
\hline
\end{tabular}

\subsection{Computational Time}

DTW-NN is computationally intensive as the DTW distance must be calculated between the ongoing batch, $\mathbf{X}$, and each batch in the reference database $\mathbf{Y}_{i}$ repeatedly for each new sample in the ongoing batch. However, in an online implementation it is only necessary to add a new row to the local distance matrices $\mathbf{C}$ and accumulated distance matrices as each new sample of $\mathbf{X}$ is obtained, 
rather than re-calculate the entire local distance matrices. The analysis was performed using the statistical software R on a 2014 laptop with an Intel quadcore processor $(2.70 \mathrm{GHz}, 2701 \mathrm{Mhz}, 8$ logical processors, $8 \mathrm{~GB}$ RAM). DTW was computed using the DTW R package [26]. Training the DTW-NN (calculating variable weights, DTW distance between reference batches throughout their durations, and control limits) took roughly 30 seconds. The elapsed real time for computing the online DTW distances between one new batch, and the 50 reference batches throughout the duration of the new batch was 0.7 seconds. To do this for all 2620 batches in one of the four datasets, parallel processing using three cores resulted in an elapsed real time of 10.1 minutes.

\section{Conclusion}

We have presented a novel data-driven online batch process monitoring tool, DTW-NN. This method was applied to four extensive datasets from a simulated penicillin production, and shown to have several advantages over the benchmark MPCA approach. DTW-NN can effectively deal with clustering of batches, and is intuitively simple in providing a direct similarity measure between an ongoing batch and previously observed NOC batches. In addition, the identification of the $k$ nearest NOC batches provides the basis for an easy to interpret visualisation tool for process operators, enabling online comparison between the present batch and its most similar predecessors. A representative database of NOC batches is required for DTW-NN. As new NOC batches are completed, they may be added to the reference database which would lead to improvements in monitoring performance. In order to scale up DTW-NN to be efficient with searching a reference database of thousands or hundreds of thousands of batches, further research may be needed, for example making use of lower bounding and other speed-up techniques [27, 28. However, DTW-NN as presented in this work may be readily implemented for effectively monitoring an ongoing batch in relation to a database of hundreds of past batches. 


\section{Acknowledgements}

This research is partially funded by BIOPRO (www.biopro.nu) which is financed by the European Regional Development Fund (ERDF), Region Zealand (Denmark) and BIOPRO partners. The authors would like to thank Anders Stockmarr for constructive criticism of the manuscript.

\section{References}

[1] P. Nomikos, J. F. MacGregor, Monitoring Batch Processes Using Multiway Principal Component Analysis, AIChE Journal 40 (1994) 1361-1375.

[2] S. Wold, N. Kettaneh, H. Fridén, A. Holmberg, Modelling and diagnostics of batch processes and analogous kinetic experiments, Chemometrics and Intelligent Laboratory Systems 44 (1998) 331-340.

[3] J. A. Westerhuis, T. Kourti, J. F. MacGregor, Comparing alternative approaches for multivariate statistical analysis of batch process data, Journal of Chemometrics 13 (1999) 397-413.

[4] D. Neogi, C. E. Schlags, Multivariate statistical analysis of an emulsion batch process, Industrial and Engineering Chemistry Research 37 (1998) 3971-3979.

[5] S. García-Muñoz, T. Kourti, J. F. MacGregor, Troubleshooting of an Industrial Batch Process Using Multivariate Methods, Industrial \& Engineering Chemistry Research (2003) 3592-3601.

[6] N. P. V. Nielsen, J. M. Carstensen, J. Smedsgaard, Aligning of single and multiple wavelength chromatographic profiles for chemometric data analysis using correlation optimised warping, Journal of Chromatography A 805 (1998) 17-35.

[7] M. Fransson, S. Folestad, Real-time alignment of batch process data using COW for on-line process monitoring, Chemometrics and Intelligent Laboratory Systems 84 (2006) 56-61. 
[8] A. Kassidas, J. F. MacGregor, P. A. Taylor, Synchronization of batch trajectories using dynamic time warping, AIChE Journal 44 (1998) 864875.

[9] J. M. González-Martínez, A. Ferrer, J. A. Westerhuis, Real-time synchronization of batch trajectories for on-line multivariate statistical process control using Dynamic Time Warping, Chemometrics and Intelligent Laboratory Systems 105 (2011) 195-206.

[10] J. M. González-Martínez, O. E. De Noord, A. Ferrer, Multisynchro: A novel approach for batch synchronization in scenarios of multiple asynchronisms, Journal of Chemometrics 28 (2014) 462-475.

[11] R. Rendall, B. Lu, I. Castillo, S.-T. Chin, L. H. Chiang, M. S. Reis, A unifying and integrated framework for feature oriented analysis of batch processes, Industrial \& Engineering Chemistry Research 56 (2017) 85908605.

[12] H. Sakoe, S. Chiba, Dynamic Programming Algorithm Optimization for Spoken Word Recognition, IEEE Transactions on Acoustics, Speech, and Signal Processing 26 (1978) 43-49.

[13] A. Mueen, E. Keogh, Extracting Optimal Performance from Dynamic Time Warping, in: Proceedings of the 22nd ACM SIGKDD International Conference on Knowledge Discovery and Data Mining - KDD '16, ACM Press, 2016, pp. 2129-2130. URL: http://dl.acm.org/citation. cfm?doid=2939672.2945383, doi $10.1145 / 2939672.2945383$.

[14] Q. P. He, J. Wang, Fault detection using the k-nearest neighbor rule for semiconductor manufacturing processes, IEEE Transactions on Semiconductor Manufacturing 20 (2007) 345-354.

[15] X. Wang, A. Mueen, H. Ding, G. Trajcevski, P. Scheuermann, E. Keogh, Experimental comparison of representation methods and distance measures 
for time series data, Data Mining and Knowledge Discovery 26 (2013) 275309.

[16] J. Van Impe, G. Gins, An extensive reference dataset for fault detection and identification in batch processes, Chemometrics and Intelligent Laboratory Systems 148 (2015) 20-31.

[17] T. Hastie, R. Tibshirani, J. Friedman, The Elements of Statistical Learning, volume 1, Springer New York, 2001. URL: http: //www.springerlink.com/index/10.1007/b94608 doi:10.1007/b94608 arXiv: arXiv: 1011.1669v3.

[18] H. J. Ramaker, E. N. M. Van Sprang, J. A. Westerhuis, A. K. Smilde, Dynamic time warping of spectroscopic BATCH data, Analytica Chimica Acta 498 (2003) 133-153.

[19] S. García-Muñoz, M. Polizzi, A. Prpich, C. Strain, A. Lalonde, V. Negron, Experiences in batch trajectory alignment for pharmaceutical process improvement through multivariate latent variable modelling, Journal of Process Control 21 (2011) 1370-1377.

[20] M. Spooner, D. Kold, M. Kulahci, Selecting local constraint for alignment of batch process data with dynamic time warping, Chemometrics and Intelligent Laboratory Systems 167 (2017) 161-170.

[21] L. Kaufman, P. J. Rousseeuw, Finding groups in data: an introduction to cluster analysis, Wiley, New York, 1990. doi:doi:10.1002/ 9780470316801.fmatter.

[22] P. Nomikos, J. F. MacGregor, Multivariate SPC charts for monitoring batch processes, Technometrics 37 (1995) 41-59.

[23] T. J. Rato, R. Rendall, V. Gomes, S. T. Chin, L. H. Chiang, P. M. Saraiva, M. S. Reis, A Systematic Methodology for Comparing Batch Process Monitoring Methods: Part I-Assessing Detection Strength, Industrial and Engineering Chemistry Research 55 (2016) 5342-5358. 
[24] D. J. Louwerse, A. K. Smilde, Multivariate statistical process control of batch processes based on three-way models, Chemical Engineering Science 55 (2000) 1225-1235.

[25] G. Birol, C. Ündey, A. Çinar, A modular simulation package for fed-batch fermentation: Penicillin production, Computers and Chemical Engineering 26 (2002) 1553-1565.

[26] T. Giorgino, Computing and Visualizing Dynamic Time Warping Alignments in R : The dtw Package, Journal of Statistical Software 31 (2009) $1-24$.

[27] E. Keogh, C. A. Ratanamahatana, Exact indexing of dynamic time warping, Knowledge and Information Systems 7 (2005) 358-386.

[28] T. Rakthanmanon, B. Campana, A. Mueen, G. Batista, B. Westover, Q. Zhu, J. Zakaria, E. Keogh, Searching and mining trillions of time series subsequences under dynamic time warping, Proceedings of the 18th ACM SIGKDD international conference on Knowledge discovery and data mining - KDD '12 (2012) 262. 